\title{
Linkage of rheumatoid arthritis to the candidate gene NRAMP1 on 2q35
}

\author{
Marie-Anne Shaw, David Clayton, Sara E Atkinson, Hazel Williams, Nancy Miller, Dean \\ Sibthorpe, Jenefer M Blackwell
}

\begin{abstract}
The macrophage resistance gene NRAMP1 regulates priming/activation of macrophages for enhanced TNF $\alpha, I L 1 \beta$, and MHC class II expression. Since all of these functions are of potential importance in the induction or maintenance or both of autoimmune disease, samples from the Arthritis and Rheumatism Council's repository of multicase rheumatoid arthritis families were typed for a dinucleotide repeat in the NRAMP1 promoter region and four other 2 q34 (TNP1) or 2q35 (IL8R, VIL1, DES) marker genes. Identity by descent (IBD) sib pair analysis using a three locus haplotype NRAMP1IL8RB-VIL1, or NRAMP1 alone, provided preliminary evidence (maximum lod score $=1.01, p=0.024$ ) for a gene in this region contributing to susceptibility to rheumatoid arthritis. Candidacy for NRAMP1 as the disease susceptibility gene was supported by a significant bias $(p=0.048)$ towards transmission of the NRAMP1 promoter region allele 3 in affected offspring.

$(\mathcal{O}$ Med Genet 1996;33:672-677)
\end{abstract}

Key words: linkage; rheumatoid arthritis; NRAMP1.

Two questions of fundamental importance in rheumatoid arthritis are (1) which genes confer disease susceptibility, ${ }^{1}$ and (2) does an infectious agent trigger disease ${ }^{2}$ Epidemiological data suggest a two locus multiplicative model for genetic susceptibility, ${ }^{3}$ to which HLA-DR has been estimated to contribute $37 \%{ }^{4}$ The major gene(s) accounting for the remaining $63 \%$ has yet to be identified.

A plausible candidate for non-MHC gene regulation of early induction of rheumatoid arthritis is the human homologue, NRAMP1, for the murine macrophage resistance gene Ity/ Lsh/Bcg (Nramp1). Ity/Lsh/Bcg was identified as a single gene controlling recessive susceptibility to the intramacrophage pathogens Salmonella typhimurium, Leishmania donovani, and Mycobacterium bovis BCG, and was mapped to the distal end of mouse chromosome $1 .^{5-7} \mathrm{~A}$ candidate for $I t y / L s h / B c g$ was identified by positional cloning, ${ }^{8}$ a full length cDNA sequence obtained, ${ }^{9}$ and its candidacy confirmed by functional analysis of transfected macrophage cell lines. ${ }^{10}$ The gene has been renamed Nramp1, the Natural resistance associated macrophage protein. ${ }^{8}$ Through a region of conserved synteny on chromosome $2 \mathrm{q} 35$, human NRAMP1 has been sequenced ${ }^{11}$ and detailed physical and genetic mapping of the region undertaken. ${ }^{12}$ This work also showed that the IL8 receptor gene cluster, IL8RA, IL8RB, and the pseudogene IL8RBP, lies in close proximity $(-130 \mathrm{~kb}$ distal $)$ to the NRAMP1 gene.

Functional studies indicate that Nramp1 regulates, and acts early in the pathway to, macrophage priming/activation for antimicrobial activity. ${ }^{5-7}$ The gene therefore has many pleiotropic effects on macrophage function, including differential upregulation of the early response gene $\mathrm{KC}$, a neutrophil chemoattractant belonging to the $\mathrm{C}-\mathrm{X}-\mathrm{C}$ family of small cytokines, as well as TNF $\alpha$, IL $1 \beta$, inducible nitric oxide synthase, and MHC class II expression, leading to higher levels in resistant macrophages. All of these functions are of potential importance in the induction or maintenance, or both, of autoimmune disease. Hence, an animal resistant to intracellular pathogens may be susceptible to autoimmune disease. A number of agents may trigger Nramp1 regulated macrophage responses, including interferon $\gamma$, bacterial lipopolysaccharide (LPS), and mycobacterial lipoarabinomannan (LAM). A role for NRAMP1 in rheumatoid arthritis would therefore also be consistent with evidence for a bacterial/ mycobacterial aetiology, ${ }^{213}$ either as a trigger for autoimmunity or as an agent for the perpetuation of disease.

To test the hypothesis that NRAMP1 influences rheumatoid arthritis, samples from the Arthritis and Rheumatism Council (ARC) Repository were typed for a dinucleotide repeat in the NRAMP1 promoter region ${ }^{11}$ and for polymorhisms at four other 2q34/35 genes, and the data analysed using an affected sib pair maximum likelihood identity by descent (IBD) method of linkage analysis and transmission disequilibrium testing.

\section{Material and methods}

Families from the ARC National Repository have been described in detail by Worthington et $a l^{14}$ and Hay et $a l^{15}$ including diagnostic criteria and family structures. In this study, 61 nuclear families from the repository were analysed, described in Worthington et $\mathrm{al}^{14}$ as families 001-046, 051-066, 075-109, 129-182, 196, $198-201,205-210,237,238,309$, and 319 . Five of these families had three affected offspring and one family had four affected offspring, giving a total of 76 affected sib pairs (although a weighting factor was applied in sib 
Table 1 Pairwise lod scores (sex averaged) for linkage between pairs of genes on 2q33-q35 from 61 pedigrees analysed using LIPED

\begin{tabular}{lrccccccc}
\hline \multicolumn{1}{c}{ Recombination fraction } \\
\cline { 2 - 8 } & \multicolumn{1}{c}{0} & 0.001 & 0.01 & 0.05 & 0.1 & 0.2 & 0.3 & 0.4 \\
\hline TNP1-NRAMP1 & -98.78 & -2.28 & -0.32 & 0.95 & 1.34 & 1.29 & 0.81 & 0.26 \\
NRAMP1-IL8R & 3.87 & 3.87 & 3.80 & 3.48 & 3.02 & 2.05 & 1.09 & 0.31 \\
IL8R-VIL1 & 4.04 & 4.03 & 3.93 & 3.47 & 2.90 & 1.82 & 0.91 & 0.25 \\
VIL1-DES & -94.55 & 3.03 & 3.94 & 4.16 & 3.81 & 2.71 & 1.51 & 0.50 \\
\hline
\end{tabular}

pair analyses for families with multiple sibs, reducing the effective number of sib pairs contributing to the analysis to 68 , see below). Nineteen sib pairs had both parents typed, 10 sib pairs had one parent typed, and 32 had no parental DNA available for typing. The total number of DNA samples available for typing for 2 q34/35 genetic polymorphisms was 318 , 129 affected and 189 unaffected.

Samples from all family members were included in typing for a repeat polymorphism immediately $5^{\prime}$ of position $-594 \mathrm{bp}$ in the NRAMP1 promoter region. ${ }^{11}$ Four variants occur within a possible enhancer element containing the Z-DNA forming dinucleotide repeat originally sequenced as $\mathrm{t}(\mathrm{gt})_{5} \mathrm{ac}(\mathrm{gt})_{5} \mathrm{ac}(\mathrm{gt})_{9} \mathrm{~g}$. Alleles 1,2 , and 3 vary in repeat number $(11,10,9$ repeats, respectively) for the larger array of gt repeats. Allele 4 carries the sequence $t(g t)_{5} a c(g t)_{10} g$. All samples were also included in typing for a new restriction fragment length polymorphism (RFLP) in IL8RB, ${ }^{12}$ and for RFLPs in genes proximal (TNP1, 2q34) and distal (VIL1 and DES, 2q35) to NRAMP1 and IL8R. ${ }^{16}$ The gene order is known to be TNP1-NRAMP1IL8RA-IL8RB-VIL1-DES. The majority of samples provided a scorable genotype. Linkage between these genes was analysed by LIPED, ${ }^{17}$ using previously reported ${ }^{12}{ }^{16}$ gene frequencies: TNP1 A1 $=0.366$ A2 $=0.634 ;$ IL8R A $1=0.314$ $\mathrm{A} 2=0.686 ; \mathrm{VIL} 1 \mathrm{~A} 1=0.672 \mathrm{~A} 2=0.328$; and DES A1 $=0.659$ A2 $=0.341$. NRAMP1 allele frequencies were those calculated by SPLINK (see below) in this analysis: $\mathrm{Al}=0.001$ A2 $=0.246$ A3 $=0.753$ A4 $=0.001$.

Linkage between markers and disease susceptibility was analysed by the affected sib pair method, using the maximum likelihood identity by descent (IBD) method pioneered by Risch $^{18}{ }^{19}$ and later extended by Holmans ${ }^{20}$ and Holmans and Clayton ${ }^{21}$ to allow for uncertainty of IBD assignment owing to unknown phase of marker haplotypes and to missing or incomplete parental genotype data. The statis- tical method involves the use of maximum likelihood to estimate the IBD sharing probabilities and haplotype frequencies simultaneously, and is implemented in the computer program SPLINK (available from the second author). In the IBD analyses, affected sib trios were dealt with by treating each trio as the three independent pairwise comparisons, but giving each comparison a weight of only $2 / 3$ in the analysis. ${ }^{22}$ Similarly the six possible pairwise comparisons of the affected 4-tuple were each given a weight of $1 / 2$. This is a conservative strategy (P Holmans, D Clayton, unpublished observation). For each marker locus examined, the transmission of individual alleles from heterozygous parents to affected offspring was examined using the transmission disequilibrium test. ${ }^{2324}$

\section{Results}

USE OF A THREE LOCUS HAPLOTYPE

Maximum lod scores for linkage between NRAMP1-IL8R and IL8R-VIL1 occurred at a recombination fraction of 0 (table 1 ), indicating that there were no recombinants between these markers in the 61 rheumatoid arthritis families. A haplotype for these three loci was therefore used in the sib pair analyses, increasing the heterozygosity from 0.371 (NRAMP1 alone) to 0.766 . Zero recombination between these markers is consistent with the physical NRAMP1-VIL1 interval of $155 \mathrm{~kb}$ and IL8RVIL1 interval of $10-30 \mathrm{~kb} .^{12}$

SIB PAIR ANALYSIS

The results of the IBD analyses for all markers are summarised in table 2. IBD sharing probabilities (0:1:2 alleles IBD) for the three locus haplotype, estimated using the maximum likelihood method, ${ }^{18}{ }^{19}$ were 0.118:0.500:0.382 giving a likelihood ratio chi-squared test statistic of 4.63. This provides a maximum lod score (MLS) of 1.01 which corresponds asymptotically to statistical significance at the $p=0.024$ level. ${ }^{20}$ The contributions which individual families make to these probabilities are represented diagramatically in fig 1 . Affected sib pairs whose IBD status is known exactly plot at the vertices of the triangles. The plotting positions of pairs calculated using maximum likelihoods reflect the relative probabilities of their assignments. In the left hand figure, the plotting positions for the probabilistic IBD assignments assume the null hypothesis (no linkage, $0.25: 0.5: 0.25)$. This plot represents

Table 2 Summary of IBD sib pair analysis using SPLINK. After weighting, the 76 affected sib pairs equated to 65-68 independent sib pairs contributing to the analysis for different genes. Effective sample size is calculated by SPLINK as the equivalent number of fully informative sib pairs. $\chi^{2}=$ likelihood ratio chi-squared test statistic. MLS $=$ maximum lod score, $p=$ probability, PIC = polymorphic information content

\begin{tabular}{|c|c|c|c|c|c|c|c|}
\hline & \multicolumn{3}{|c|}{ Estimated IBD sharing probabilities } & \multirow{2}{*}{$\begin{array}{l}\text { Effective } \\
\text { sample size }\end{array}$} & \multirow[b]{2}{*}{$\chi^{2}$} & \multirow[b]{2}{*}{$M L S$} & \multirow[b]{2}{*}{$p$} \\
\hline & 0 -ibd & 1 -ibd & 2 -ibd & & & & \\
\hline TNP1 & 0.250 & 0.500 & 0.250 & 19 & 0 & 0 & 0 \\
\hline NRAMP1 & 0.127 & 0.420 & 0.453 & 13 & 4.64 & 1.01 & 0.024 \\
\hline NRAMP1-IL8R-VIL1 & 0.118 & 0.500 & 0.382 & 27 & 4.63 & 1.01 & 0.024 \\
\hline DES & 0.182 & 0.500 & 0.318 & 19 & 0.90 & 0.20 & 0.171 \\
\hline
\end{tabular}

NRAMP1: heterozygosity $=0.371$, PIC score $=0.302$.

NRAMP1-IL8R-VIL1: heterozygosity $=0.766$, PIC score $=0.740$.

TNP1: heterozygosity $=0.487$, PIC score $=0.368$.

DES: heterozygosity $=0.437$, PIC score $=0.342$. 


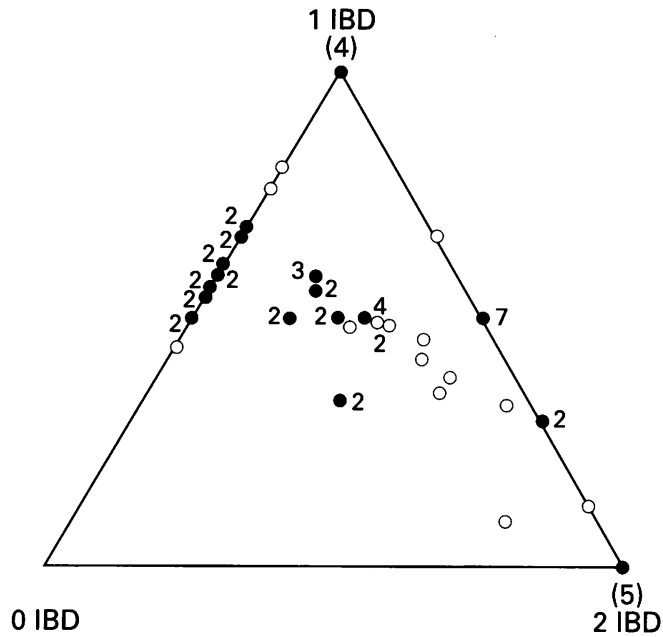

Null hypothesis

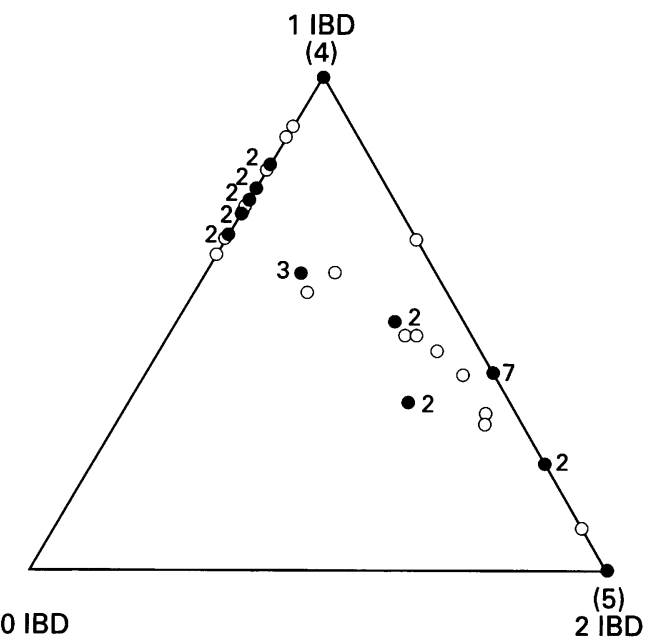

Maximum likelihood

Figure 1 Contributions of individual families to IBD haplotype sharing probabilities. An affected sib pair whose IBD status is known exactly is plotted at the vertices of the triangles. The plotting position of other pairs depends on the relative probabilities of their assignments. All 59 families informative for the three locus haplotype contribute to the maximum lod score. Open circles indicate single sib pairs while closed circles indicate the contribution of more than one sib pair. In the latter case the number of pairs is shown alongside the plotting point.

Table 3 NRAMP1-IL8R-VIL1 haplotype frequencies derived from 59 families/66 sib pair comparisons using SPLINK

\begin{tabular}{lrlc}
\hline Haplotype & Frequency & Haplotype & Frequency \\
\hline $1-1-1$ & $<0.001$ & $1-1-2$ & $<0.001$ \\
$2-1-1$ & 0.010 & $2-1-3$ & 0.017 \\
$3-1-1$ & 0.133 & $3-1-2$ & 0.077 \\
$1-2-1$ & $<0.001$ & $1-2-2$ & $<0.001$ \\
$2-2-1$ & 0.134 & $2-2-2$ & 0.083 \\
$3-2-1$ & 0.406 & $3-2-2$ & 0.138 \\
\hline
\end{tabular}

the evidence on which the significance test is based. The right hand figure uses the assignment probabilities at the final maximum likelihood estimate of the sharing probabilities. Summation of these assignments provides the maximum likelihood estimates. None of the 10 fully informative families showed 0-IBD for the NRAMP1-IL8R-VIL1 haplotype. The positions for the 49 families obtained using probability assignments depict the clear balance of probability away from the null hypothesis. In this maximum likelihood analysis, the marker haplotype frequencies are estimated internally. This guards against errors which may follow from misspecification by taking haplotype frequencies from inappropriate populations. The fact that these frequencies are estimates rather than known constants is allowed for in the likelihood ratio tests. The final estimates of the NRAMP1-IL8R-VIL1 haplotype frequencies are shown in table 3.

Table 4 Transmission disequilibrium testing

\begin{tabular}{lllll}
\hline & $\begin{array}{l}\text { No of transmissions from } \\
\text { heterozygous parents }\end{array}$ & No of families & Allele & $\begin{array}{l}\text { No of transmissions } \\
\text { for each allele }\end{array}$ \\
\hline TNP1 & 54 & 38 & 1 & 22 \\
& & & 2 & 32 \\
NRAMP1 & 31 & 25 & 2 & $10 \chi^{2}=3.9$ \\
& & & 3 & $21 \mathrm{p}=0.048$ \\
IL8R & 21 & 15 & 1 & 12 \\
VIL1 & 38 & 30 & 2 & 9 \\
& & & 1 & 19 \\
DES & 52 & 35 & 2 & 19 \\
& & & 2 & 21 \\
\hline
\end{tabular}

When the IBD analysis was repeated for NRAMP1 alone (table 2), the maximum lod estimates of the IBD sharing probabilities were (0.127:0.420:0.453), again giving a likelihood ratio chi-squared test statistic of 4.64 and a MLS of 1.01. Hence, even without the additional polymorphic information content which the haplotype provides, a positive association between NRAMP1 and susceptibility to rheumatoid arthritis is observed. In contrast, no linkage between rheumatoid arthritis and the markers either proximal (TNP1) or distal (DES) to the three locus haplotype (table 2) was observed, even though the polymorphic information content for these markers was higher than for the NRAMP1 promoter region polymorphism.

TRANSMISSION TESTING

While NRAMP1 is a candidate locus, and linkage has been established to $2 \mathrm{q} 35$, it is possible that another gene in the region of the three locus haplotype is responsible for susceptibility. To address this issue, transmission of alleles from heterozygous parents was examined for the five 2q34/35 genes using the same set of families (table 4). Since problems may arise in transmission testing where parental genotype information is incomplete, ${ }^{25}$ only matings where parental genotypes were conclusively assigned were used in the analysis. In the majority of cases this meant families where both parents were available for typing. For NRAMP1, two families were included where the heterozygous parental genotype was deduced on the basis of genotypes of offspring from the extended family, that is, unaffected as well as affected offspring were available. In both cases, there was only one possible parental genotype which could be assigned, and this could be done without reference to the affected person being tested for transmission disequilibrium. The only significant deviation from the expected 1:1 transmission of the two alleles to affected sibs under the null hypothesis was obtained for NRAMP1, where allele 3 was 
Table 5 Risk of rheumatoid arthritis in sib sharing 0, 1, or 2 NRAMP-IL8R-VIL1 $2 q 35$ or HLA haplotypes IBD with an affected sib

\begin{tabular}{lll}
\hline & $\begin{array}{l}\text { NRAMP1-IL8R-VIL1 } \\
(\%)\end{array}$ & $\begin{array}{l}\text { HLA } \\
(\%)\end{array}$ \\
\hline 0-IBD & 1.8 & 2.3 \\
1-IBD & 3.9 & 3.5 \\
2-IBD & 6.0 & 6.2 \\
\hline
\end{tabular}

transmitted to affected children in preference to allele 2 (chi-squared $=3.9, \mathrm{p}=0.048$ ). In testing for transmission disequilibrium, multiple independent tests have been carried out Hence, it could be argued that a multiple testing correction factor should be applied. Multiplying our $p$ values by the number of tests performed $(2$ alleles $\times 5$ loci $=10)$ would clearly remove all significance from the NRAMP1 TDT allelic association in this small dataset. However, as Copeman et $a l^{26}$ argue, if we had tested only the loci showing independent evidence of linkage (NRAMP1), a correction factor of only 2 (=2 alleles, 1 locus) could be applied. Since we could also argue that a one tailed test could be applied in comparing alleles 2 versus 3 , that is, that we would expect a one way skew towards allele 3 in affected offspring on functional grounds (S Searle, J M Blackwell, unpublished data, see Discussion), our original $\mathrm{p}$ value for NRAMP1 TDT could be halved. Multiplying by 2 would then retain the TDT for NRAMP1 within the $5 \%$ significance level.

RELATIVE IMPORTANCE OF A GENE AT 2Q35 Using the sib recurrence risk of $3.9 \%,{ }^{4}$ the risk of disease to sibs sharing 0,1 , or 2 NRAMP1IL8R-VIL1 haplotypes IBD with an affected sib were calculated and compared with previous estimates ${ }^{27}$ for HLA haplotype sharing (table 5). The value of $6.0 \%$ for a sib sharing two NRAMP1 haplotypes IBD is comparable to the risk of $6.2 \%$ calculated for HLA. ${ }^{27}$ The value of $2.3 \%$ for 0 -IBD HLA haplotypes, which is greater than the observed population prevalence of $1 \%{ }^{28}$ for sibs sharing 0-IBD HLA haplotypes, is itself indicative of other factors operating, such as additional genetic loci. The degree of familial clustering is the ratio of the risk to the sibs of affecteds and the population prevalence: $=3.9$ for rheumatoid arthritis. ${ }^{29}$ The relative contribution of a locus to this total is the ratio of expected to observed sharing no alleles IBD ${ }^{26}$ : $0.25 / 0.118=2.1$ (a) for the NRAMP1-IL8R-VIL1 haplotype, 0.25/0.127 $=2.0$ for NRAMP1 alone, and 0.25/0.15 = 1.7 (b). for HLA. Using the additive model of Risch, ${ }^{30}$ where either NRAMP1 or HLA haplotypes could confer susceptibility, a total contribution $[(a-1)+(b-1)+1]$ of 2.8 $(1.1+0.7+1)$ is obtained. A multiplicative model $(\mathrm{a} \times \mathrm{b})$, where both loci operate, gives a total contribution of $3.6(2.1 \times 1.7)$. Whichever is the case, it is clear that NRAMP1 (or a tightly linked gene) could make a significant contribution to disease susceptibility, comparable to that observed previously for HLA.

\section{Discussion}

Results of the IBD sib pair analysis presented here provide evidence (MLS $=1.01, \mathrm{p}=0.024$ ) for the presence of a gene contributing to susceptibility to rheumatoid arthritis in the region of the NRAMP1-IL8R-VIL1 haplotype on human chromosome $2 \mathrm{q} 35$. Since this region contained an a priori candidate gene (NRAMP1) for disease susceptibility on functional grounds, there was no multiple testing problem. We have performed a single test of the null hypothesis of no linkage to $2 \mathrm{q} 35$, with a resulting pointwise nominal significance level ${ }^{31}$ of $2.4 \%$. Since this region was not identified as part of a genome wide search, the more stringent criteria for genome wide significance levels suggested by Lander and Kruglyak $^{31}$ do not apply. Candidacy for NRAMP1 as the disease susceptibility gene within 2q35 was supported by (1) IBD analysis for NRAMP1 alone providing an equivalent MLS of 1.01, and (2) a significant bias $(p=0.048)$ towards transmission of the NRAMP1 promoter region allele 3 in affected offspring. The latter observation suggests that the NRAMP1 promoter region polymorphism itself may be the disease associated genetic defect in these families. Although a role for IL8R genes cannot be discounted, NRAMP1 itself does provide the more plausible functional candidate for non-MHC control of susceptibility to rheumatoid arthritis. Its pleiotropic effects ${ }^{5-7}$ include roles in cytokine production, in particular TNF $\alpha$ and IL $1 \beta$, and in MHC class II antigen presentation to CD $4^{+}$ $\mathrm{T}$ cells. There is evidence that macrophages are directly involved in the inflammatory process in rheumatoid arthritis, and that $\mathrm{TNF} \alpha$ plays a major role in autoimmune disease. IL1 $\beta$ and TNF $\alpha$ are both expressed by synovial tissue macrophages in rheumatoid arthritis. ${ }^{32}$ GMCSF is also produced in large quantities by activated macrophages in the synovium, and acts to induce IL-1 $\beta$ production and enhance class II expression. ${ }^{33}$ Hence, there is good evidence that the activation phenotypes associated with NRAMP1 regulated macrophage activation could contribute directly to the inflammatory response and pathology associated with rheumatoid arthritis. Results of continuing research in our laboratory (S Searle, J $M$ Blackwell, unpublished data) analysing promoter function in a reporter gene system indicate that allele 3 of the NRAMP1 promoter region polymorphism drives NRAMP1 expression more efficiently than allele 2 . This would be consistent with overexpression of the NRAMP1 gene, and hence hyperactivation of macrophages, contributing to disease susceptibility. Although additional functional polymorphisms/mutations in NRAMP1 may be found, there is good evidence that this promoter region polymorphism may represent the functional $2 \mathrm{q} 35$ polymorphism contributing to disease susceptibility in families analysed here.

In previous studies, polymorphic HLA haplotypes have been shown to contribute to disease susceptibility in rheumatoid arthritis, either through the direct role of MHC class II molecules in presenting antigen to autoim- 
mune $\mathrm{T}$ cells or through linkage disequilibrium with polymorphic elements regulating TNF $\alpha$ production. ${ }^{34}$ The possible interaction between NRAMP1 and MHC class II regulation of disease susceptibility is of particular interest. One hypothesis for polygenic control in rheumatoid arthritis is that non-MHC genes determine susceptibility to disease per se and act early in the induction phase of autoimmunity, whereas HLA determines disease severity through modulation of $\mathrm{T}$ cell responses to particular antigens. ${ }^{35} 36$ This parallels observations in the mouse where Nramp1 influences the early macrophage response to infection, and hence susceptibility to disease per se, while polymorphism in class II molecules at the mouse $\mathrm{H}-2$ locus influences disease severity. ${ }^{37}$ For control of infection, the best possible interaction between genes occurs when an Nramp1 resistant mouse carries an $\mathrm{H}-2$ curing haplotype, leading to enhanced antigen presentation to protective interferon $\gamma$ generating $\mathrm{CD}_{4}{ }^{+} \mathrm{T}$ helper 1 cells. In autoimmune disease, this might represent the worst possible interaction. $\mathrm{T}$ cells, particularly $\mathrm{CD} 4^{+}$cells, contribute to pathology in rheumatoid arthritis. The involvement of cells recognising antigen in association with HLA class II molecules fits well with the association of rheumatoid arthritis with particular DR types ${ }^{36}$ and also points to a significant role for antigen presenting cells such as macrophages. Further analysis of the interaction between DR and NRAMP1 in determining RA susceptibility will be of particular interest.

NRAMP1 as a candidate for susceptibility to rheumatoid arthritis is also interesting in relation to a bacterial/mycobacterial aetiology for the disease. The gene was first identified for its role in controlling resistance to intramacrophage pathogens. Many arthritic diseases have been shown to have intracellular pathogens as triggers, and the hypothesis that rheumatoid arthritis is a slow bacterial infection with autoimmunity a secondary consequence has not been disproved. ${ }^{13}$ In the case of rheumatoid arthritis, no self antigen has been shown to be associated with the initiation of disease. Mycobacteria contain inducers of TNF- $\alpha$, the most potent of which is LAM, ${ }^{38}$ while organisms associated with reactive arthritis have LPS which can be identified in affected joints. ${ }^{39}$ Both LAM and LPS are important triggers of Nramp1 regulated macrophage activation. ${ }^{6} 7$ Support for a role of mycobacteria in arthritis has come from the use of Lewis rats which are susceptible to adjuvant induced arthritis and respond particularly to the mycobacterial $65 \mathrm{kDa}$ hsp. Indeed, arthritis is inducible by a single $T$ cell clone recognising the non-conserved $180-188$ region of mycobacterial hsp65. ${ }^{40} \mathrm{~T}$ cells isolated from the synovial fluid of patients have been found to give a localised response to both conserved and non-conserved mycobacterial hsp65 epitopes. ${ }^{41}$ The reactivity against non-conserved epitopes of hsp65 cannot be accounted for by cross reactions with the human homologue. Again, the interaction between NRAMP1 and MHC class II molecules in directing the $\mathrm{T}$ cell response to bacterial/mycobacterial antigens may be of particular importance in initiation and maintenance of disease.

The primary function of NRAMP1 remains unknown. While its molecular structure is compatible with a transport function, the presence of an $\mathrm{SH} 3$ binding domain at the $\mathrm{N}$-terminus of the molecule points to a role in signal transduction. ${ }^{9}$ In the murine system, the final effector mechanism for antimicrobial activity relies on induction of nitric oxide synthase and generation of nitric oxide. ${ }^{7}$ This, or any one or combination of the multiple pleiotropic effects of the gene, would be sufficient to account for a role for NRAMP1 in regulating autoimmune disease.

In this study, only $47 \%$ of families had one $(16 \%)$ or both $(31 \%)$ parents of affected sibs available for study, reducing the power of our analysis in terms of equivalence to fully informative affected sib pairs (table 2). This is a general problem in late onset diseases. Our results are therefore preliminary, and require confirmation in additional datasets before the real contribution of NRAMP1 to disease susceptibility in rheumatoid arthritis is known. Functional studies analysing NRAMP1 expression in synovial tissue macrophages would also be of interest, as well as studies comparing activation phenotypes in macrophages from people bearing different NRAMP1 genotypes. These continuing studies of NRAMP1 in rheumatoid arthritis patients and families provide an exciting basis to further genetic and functional characterisation of disease susceptibility.

This work was supported by grants from the Arthritis and Rheumatism Council and the Wellcome Trust. We would like to thank Dr Bill Ollier, ARC Epidemiology Research Unit, Manchester University, for his help in making material available from the ARC Repository.

1 Wordsworth P, Bell J. Polygenic susceptibility in rheumatoid arthritis. Ann Rheum Dis 1991;50:343-6.

2 Silman AJ. Is rheumatoid arthritis an infectious disease? BMF 1991;303:200.

3 Rigby AS, Voelm L, Silman AJ. Epistatic modelling in rheumatoid arthritis: an application of the Risch theory. Genet matoid arthritis: an applica

4 Deighton CM, Walker DJ, Griffiths ID, Roberts DF. The contribution of HLA to rheumatoid arthritis. Clin Genet 1989;36:178-82.

5 Blackwell JM (Convenor, 27th Forum in Immunology). The macrophage resistance gene $\mathrm{Lsh} / \mathrm{Ity} / \mathrm{Bcg}$. Res Immunol 1989;140:767-828.

6 Blackwell JM, Roach TIA, Atkinson SE, et al. Genetic regulation of macrophage priming/activation: the Lsh gene story. Immunol Lett 1991;30:241-8.

7 Blackwell $\mathrm{JM}$, Barton $\mathrm{CH}$, White $\mathrm{JK}$, et al. Genetic regulation of leishmanial and mycobacterial infections: the $\mathrm{Lsh} / \mathrm{Ity} / \mathrm{Bcg}$ gene story continues. Immunol Lett 1994; 43:99-107.

8 Vidal SM, Malo D, Vogan K, Skamene E, Gros P. Natural resistance to infection with intracellular parasites: isolation of a candidate for $B c g$. Cell 1993;73:469-85.

9 Barton CH, White JK, Roach TIA, Blackwell JM $\mathrm{NH}_{2}$-terminal sequence of macrophage-expressed natural $\mathrm{NH}_{2}$-terminal sequence of macrophage-expressed natural resistance-associated macrophage protein ( $\mathrm{Nramp}$ ) encodes a proline/serine-rich putative $S r c$ ho

10 Barton CH, Whitehead SH, Blackwell JM. Nramp transfection transfers Ity/Lsh/Bcg-related pleiotropic effects on tion transfers $I t y / L s h / B c g$-related pleiotropic effects on macrophage activation: influence on oxidative

11 Blackwell JM, Barton CH, White JK, et al. Genomic organization and sequence of the human NRAMP gene: identification and mapping of a promoter region polymorphism. Mol Med 1995;1:194-205.

12 White JK, Shaw MA, Barton $\mathrm{CH}$, et al. Genetic and physical mapping of $2 \mathrm{q} 35$ in the region of NRAMP and IL8R genes: identification of a polymorphic repeat in exon 2 of NRAMP. Genomics 1994;24:295-302.

13 Rook G, McCulloch J. HLA-DR4, mycobacteria, heatshock proteins, and rheumatoid arthritis. Arthritis Rheum 1992;35:1409-12. 
14 Worthington J, Ollier WER, Leach MK, et al. The Arthritis and Rheumatism Council's National repository of family material: pedigrees from the first 100 rheumatoid arthritis families containing affected sibling pairs. $\mathrm{Br} f$ Rheumatol 1994;33:970-6.

15 Hay EM, Ollier WER, Silman AJ. The Arthritis and Rheumatism Council's national family material repository. $\mathrm{Br} \mathscr{f}$ Rheumatol 1993;32:443-4.

16 Shaw MA, Atkinson S, Dockrell $\mathrm{H}$, et al. An RFLP map for 2q33-q37 from multicase mycobacterial and leishmania disease families: no evidence for an $L s h / I t y / B c g$ gene homologue influencing susceptibility to leprosy. Ann Hum Genet 1993;57:251-71.

$17 \mathrm{Ott} \mathrm{J}$. Linkage analysis and family classification under heterogeneity. Ann Hum Genet 1983;47:311-20.

18 Risch N. Linkage strategies for genetically complex traits. II. The power of affected relative pairs. Am $\mathcal{f}$ Hum Genet The power of a
1990;46:229-41.

19 Risch N. Linkage strategies for genetically complex traits. III. The effect of marker polymorphism on analysis of affected relative pairs. Am f Hum Genet 1990;46:242-53.

20 Holmans P. Asymptotic properties of affected-sib-pair linkage analysis. Am $\mathcal{H}$ Hum Genet 1993;52:362-74.

21 Holmans P, Clayton D. Efficiency of typing unaffected relatives in an affected sib-pair linkage study with single locus and multiple tightly-linked markers. Am 7 Hum Genet 1995; 37:1221-32.

22 Payami H, Thomson G, Motro U, Louis EJ, Hudes E. The affected sib pair method. IV. Affected sib trios. Ann Hum Genet 1985;49:303-14.

23 Spielman RS, McGinnis RE, Ewens WJ. Transmission test for linkage disequilibrium: the insulin gene region and insulin-dependent diabetes mellitus (IDDM). Am $\mathcal{F} \mathrm{Hum}$ insulin-dependent diabet

24 Schaid DJ, Sommer SS. Comparison of statistics for candidate-gene association studies using cases and parents. Am f Hum Genet 1994;55:402-9.

25 Curtis D, Sham PC. A note on the application of the transmission disequilibrium test when a parent is missing. $A m \mathcal{F}$ Hum Genet 1995;56:811-12.

26 Copeman JB, Cucca C, Hearne CM, et al. Linkage disequilibrium mapping of a type 1 diabetes susceptibility gene (IDDM7) to chromosome 2q31-q33. Nature Genet 1995;9:80-5.

27 Rigby AS. HLA haplotype sharing in rheumatoid arthritis sibships: risk estimates in siblings. Scand $\mathcal{f}$ Rheumatol 1992;21:68-73.
28 Hazes JMW, Silman AJ. Review of UK data on rheumatic diseases-2. Rheumatoid arthritis. Br $\mathcal{f}$ Rheumatol 1990; 29:310-12.

29 Risch N. Assessing the role of HLA-linked and unlinked determinants of disease. Am $\mathcal{F}$ Hum Genet 1987;40:1-14.

30 Risch N. Linkage strategies for genetically complex traits. I. Multilocus models. Am f Hum Genet 1990;46:222-8.

31 Lander E, Kruglyak L. Genetic dissection of complex traits: guidelines for interpreting and reporting linkage results. guidelines for interpreting and

32 MacNaul KL, Hutchinson NI, Parsons JN, Bayne EK, Tocci MJ. Analysis of IL-1 and TNF-alpha gene expression in human rheumatoid synoviocytes and normal monocytes by in situ hybridization. F Immunol 1990; 145:4154-66.

33 Alvaro-Gracia JM, Zvaifler NJ, Firestein GS. Cytokines in chronic inflammatory arthritis. IV Gm-CSF mediated induction of class II MHC antigen on human monocytes: a possible role in rheumatoid arthritis. f Exp Med a possible role

34 Jacob CO. Tumour necrosis factor-alpha in autoimmunity: pretty girl or old witch? Immunol Today 1992;13:122-5.

35 Wicks I, McColl G, Harrison L. New perspectives on rheumatoid arthritis. Immunol Today 1994;15:553-6.

36 Singal DP, Green D, Reid B, Gladman DD, Buchanan WW. HLA-D region genes and rheumatoid arthritis (RA): importance of DR and DQ genes in conferring susceptibilimportance of DR and DQ genes in confe

37 Blackwell J, Freeman J, Bradley D. Influence of $\mathrm{H}-2$ complex on acquired resistance to Leishmania donovan infection in mice. Nature 1980;283:72-4.

38 Chatterjee D, Roberts $\mathrm{AD}$, Lowell K, Brennan PJ, Orme IM. Structural basis of capacity of lipoarabinomannan to induce secretion of tumor necrosis factor. Infect Immun 1992;60:1249-53.

39 Granfors K, Jalkanen S, Linberg AA, et al. Salmonella lipopolysaccharide in synovial cells from patients with reactive arthritis. Lancet 1990;335:685-8.

40 Van Eden W, Thole JE, van der Zee R, et al. Cloning of the mycobacterial epitope recognized by $\mathrm{T}$ lymphocytes in adjuvant induced arthritis. Nature 1988;331:171-3.

41 Gaston JS, Life PF, Jenner PJ, Colston MJ, Bacon PA. Recognition of a mycobacteria specific epitope in the $65-\mathrm{kD}$ heat-shock protein by synovial fluid-derived $T$ cell clones. f Exp Med 1990;171:831-41. 